\title{
Electronic Marine Animal Tagging: New Frontier in Ocean Science
}

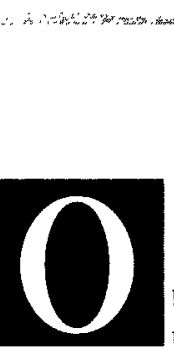

n May 14 and 15, 1998, a forum was held as part of the Aquatic Forum Series at the New England Aquarium to assess the current status of electronic marine animal telemetry tags and the next steps to advance and apply this technology to better understand the patterns of life in the oceans. The forum was sponsored by the New England Aquarium, Alfred P. Sloan Foundation, and Marine Technology Society (MTS). To coincide with this meeting, the Marine Technology Society published a special issue of the Marine Technology Society Journal on marine animal telemetry tagging (Stone and Kraus, 1998), which was distributed to participants and provided a basis for discussion.

Most participants in this forum had backgrounds in oceanography, marine animal biology and/or engineering. Participants were asked to "...explore the value, timeliness and feasibility of stimulating, designing, and organizing a period of comprehensive oceanic observation whose purpose would be to assess and explain the global distribution and abundance of marine life" (see synopsis by Bannister, this volume). One of the major recommendations that emerged from the non-fish nekton workshop held at the New England Aquarium on December 10 and 11, 1997 was to further explore the applicability of electronic marine animal tagging (see synopsis by Levi et al., this volume).

Further guidance for the tagging forum came from the summary meeting of all "The Census of the Fishes"sponsored workshops to date, held in Monterey, California on January 14 and 15, 1998 (see synopsis by Bradley, this volume). At that meeting, the challenge of this overall effort was summarized by the following statement: "Determine the patterns of animal life in the ocean and the mechanisms by which they are maintained; and determine how much life the ocean can sustain."

This challenge provided a point of departure for the New England Aquarium meeting for a series of background talks on fish, invertebrate, turtle, marine mammal and sea bird tagging. With this information as a foundation, the participants were divided into three working groups to address the following questions:
Gregory Stone, Jerry Schubel and Heather Tausig New England Aquarium - Boston, Massachusetts USA

- What is the state of technology and the availability of tags to address the challenges formulated at the Monterey meeting?

- Is there a compelling rationale for increasing the numbers of tags and the amount of tagging?

- What could tagging contribute (e.g. challenges, which taxa)?

- Where will the investment in technology development pay the biggest dividend?

- What would your ideal tag look like (e.g. animal and challenge specific)?

- What are the limiting factors for tags (e.g. satellite, acoustic, etc.)?

- What data transmission, management and capacities are needed?

The working groups provided reports in plenary, which were followed by discussions on data transmission and management, animal rights, and human training issues.

\section{Data Transmission and Management Issues}

Workshop participants recognized that there are three basic types of electronic marine animal tags currently in use today:

1. Sonic Tags are acoustic devices attached to marine animals and tracked through the water with hydrophones. Because electromagnetic energy attenuates rapidly in water, it is difficult to use radio telemetry for animals that rarely or never come to the surface. Sonic tags are useful for these animals, which include invertebrates and some fish. The major limitation is that sonic tags utilize high frequency sound and receivers must be fairly close to the tagged animals to record the signal.

2. Satellite Tags are attached to animals and are designed to transmit data to satellites via radio telemetry when the antenna of the tag comes out of the water. This is useful for all marine animals that 
surface to breathe air, such as marine mammals and turtles, and for other animals that routinely break the surface such as basking sharks, ocean sunfish and some species of tuna.

3. Archival Tags are devices attached to animals that record various data, such as position, temperature, and water depth, which are then recovered from the animal so that the archived data can be downloaded. This retrieval system works best for animals that return to predictable land sites such as nesting birds and pinnipeds, or animals that will have a high likelihood of being recaptured in the ocean, such as some tuna. Another type of archival tag separates from the animal after a period of days, weeks, or months, and floats to the surface where it then transmits data to a satellite. This is called satellite "pop-up" technology and is applicable for all kinds of marine animals.

In an effort to assess current electronic marine tagging effort, participants estimated that in the last year approximately 11,800 electronic tags were placed on various marine animals at locations throughout the world. These tags were distributed in the following groups:

\begin{tabular}{|cc|}
\hline Marine Animal & Total Tags \\
\hline Marine Mammals: & $>1,250$ \\
whales $(>100)$ & \\
dolphins $(>100)$ & \\
sirenians $(>50)$ & \\
pinnipeds $(>1,000)$ & $>10,000$ \\
\hline Marine Fish & $>300$ \\
Marine Reptiles & $>36$ \\
Marine Invertebrates & $>200$ \\
Marine Birds & \\
\hline
\end{tabular}

During discussions, it became apparent that, while many of these tags were used by a number of independent researchers, there was little or no coordination among research projects. This illustrated the current disparate nature of tagging projects throughout the world and the need for coordination. To begin with, participants felt that by simply coordinating existing efforts and comparing data we would learn more about animal biology, trophic and social interactions, and ecosystem processes. There was also strong agreement that a substantially expanded and coordinated electronic tagging program has enormous potential to significantly advance our understanding of the oceans and address the challenges issued at the Monterey meeting.

\section{Animal Rights Issues}

A representative of The Humane Society of the United States led a discussion on the ethics of tagging. The group recognized that there may be objections from certain groups to attaching electronic devices to wild animals, particularly marine mammals. The Acoustic Thermometry of Ocean Climate (ATOC) experiment in the Pacific Ocean, where sounds are generated to measure ocean temperature, was an example of an apparently sound scientific effort that may have initially gone forward without adequate consultation with groups concerned about animals. This resulted in severe objections from members of the animal rights community regarding the unknown risks to the animals.

It was also recognized that there should be a strong research and conservation component to any tagging work and that the importance of the work should outweigh any potential negative impacts on the animals. All through this process animal rights groups should be consulted.

\section{Human Training Issues}

Placing tags on marine animals and then receiving and analyzing data from these tags is a sophisticated procedure. If a larger number of tags are used for research worldwide, there will be a corresponding need to increase training of individuals to carry out this work. Much of this human resource need could be filled by graduate students and other researchers who will be funded along with the tags. Manufacturers of tags commented that they are increasingly getting inquiries from potential users who know nothing about tagging. In the past, users were more technically-oriented and had a better understanding of the technology they were using. Now, as tagging becomes more sophisticated and widespread, non-technical users want access to this technology. Manufacturers and others may need to prepare for these kinds of inquiries by offering a graduate course or formal training program.

\section{Recommendations}

The following is a list of "next step" projects and recommendations made by participants at the conclusion of the workshop. There is no rank or order to these recommendations.

- Plan and execute a demonstration project or "synoptic study" for electronic tags. Select a location and target various animal groups to test the feasibility of simultaneously tagging several marine species and monitoring their movement and behavior relative to each other and to the oceanographic features of the region. There was a strong consensus that this was 
the best way to quickly advance the concept of using electronic marine animal tagging in a multi-disciplinary and comprehensive way, and could be a prelude to larger efforts in the future. Most tagging has been of individual animals or groups of animals in isolation from their interactions with other animals and the environment. The size of a demonstration site was open to differing opinions. Some possible locations included the Gulf of Maine, Monterey Bay, North Atlantic Ocean, Southern Ocean and the Bering Sea. Whatever the location, it should be a "hot spot," or region of concentrated animal activity (e.g. nursery, migration corridor, feeding and/or breeding area), have available multiple "animal platforms" to carry tags (e.g. large pelagic fish, squid, marine mammals, etc.) and have associated physical and chemical oceanographic data available for analysis and comparison. To gain the most information on both the marine animal interactions and the use of tags, all types of tags (sonic, archival, satellite) should be considered for this project.

- Write a multi-authored paper from existing data and publications on the abundance and distribution of ocean life based on a top-down view of oceanography. This would provide a contrast to traditional oceanography that views the ocean from a "bottom-up" perspective. This review will place the use of telemetry data in perspective, since most telemetry systems work with higher level predators such as large fish, marine mammals, turtles, etc. This would also link lower trophic levels with the higher levels.

- Integrate existing databases and improve software. Before embarking on a bold new research initiative, it is important to review and summarize all existing data and obtain new or improved software. The tagging community needs to rank software and programming needs in terms of what we need today in order to achieve future goals. Geo-Locating System (GLS) software needs improvement. Evaluate all existing tags; review all animal tracks and the performance of electronic tags.

- Identify and rank animals that are good platforms for tags. Include animals whose behavior will show trophic level interactions and information on ocean processes such as currents, temperature, etc.

- Increase public outreach and education programs. It is important that the public understand and support this initiative. We will need an attention-gathering name for the project such as "Tags of Discovery" or "Turtles as Teachers" to bolster public support. The National Science Foundation-sponsored WhaleNet program at Wheelock College was cited as an example of good education and research. Aquariums also offer opportunities to expose the public to this initiative through education programs and by exhibiting tagged animals.

- Improve batteries and power sources for tags.

- Develop a website on marine animal tagging with the purpose of providing information and better defining this new discipline of oceanography.

- Move to fully utilize stranding and rehabilitation programs world-wide. This is an existing pool of opportunities for tagging animals. Aquariums and rehabilitation programs need to understand tagging in relation to conservation and research. In the United States, a workshop would need to include a partnership with the National Oceanic and Atmospheric Administration/National Marine Fisheries Service Office of Protected Resources in order to increase the use of tags with stranded animals. There would be similar or related agencies in other countries that would need to be involved if stranded animals were used.

- Evaluate the use of trained animals with tags to explore particular aspects of the ocean. What is the utility of training marine mammals to carry tags to specific areas? This differs fundamentally from tagging wild animals and learning what they do.

\section{Potential Next-Step Workshops}

Bringing interested and talented scientists and engineers together for discussions on the following topics would be an effective way to begin solving the key problems of marine animal tagging.

- Tag attachment techniques for all animals. We need better ways to attach tags to marine animals. This workshop needs to have a biomedical emphasis.

- Miniaturization of all tag components. Making tags smaller is key to advancing this science.

- Geo-Locating System (GLS) calculation of longitude/latitude. Astronomers and mathematicians will need to be involved.

- Software for data analysis and 4-dimensional displays and use. There are programming needs for analyzing data acquired by tags.

\section{Conclusion}

A program using electronic marine animal telemetry tags will help meet the challenge laid out at the beginning of this note, which was to "Determine the patterns of animal life in the ocean and the mechanisms by 
which they are maintained; and determine how much life the ocean can sustain." Placing tags on marine animals enables researchers to sense the ocean and record behavior from the animal's perspective, a fundamental shift from traditional oceanographic sampling techniques. Advances in tag engineering and signal processing within the last few years offer new opportunities to advance our understanding of animal behavior and ocean systems. The next steps and recommendations laid out in this workshop will facilitate and quicken this process.

\section{REFERENCES}

ICES, 1997: Draft ICES concept note on "The Census of the Fishes". Written by Jesse Ausubel and coordinated by Colin Bannister. Paper is available at http://phe.rockefeller.edu/fish.

Stone, G.S. and S.D. Kraus, 1998: Following the invisible: Electronic tracking of marine animals. MTS Journal, 32(1), 3-4.

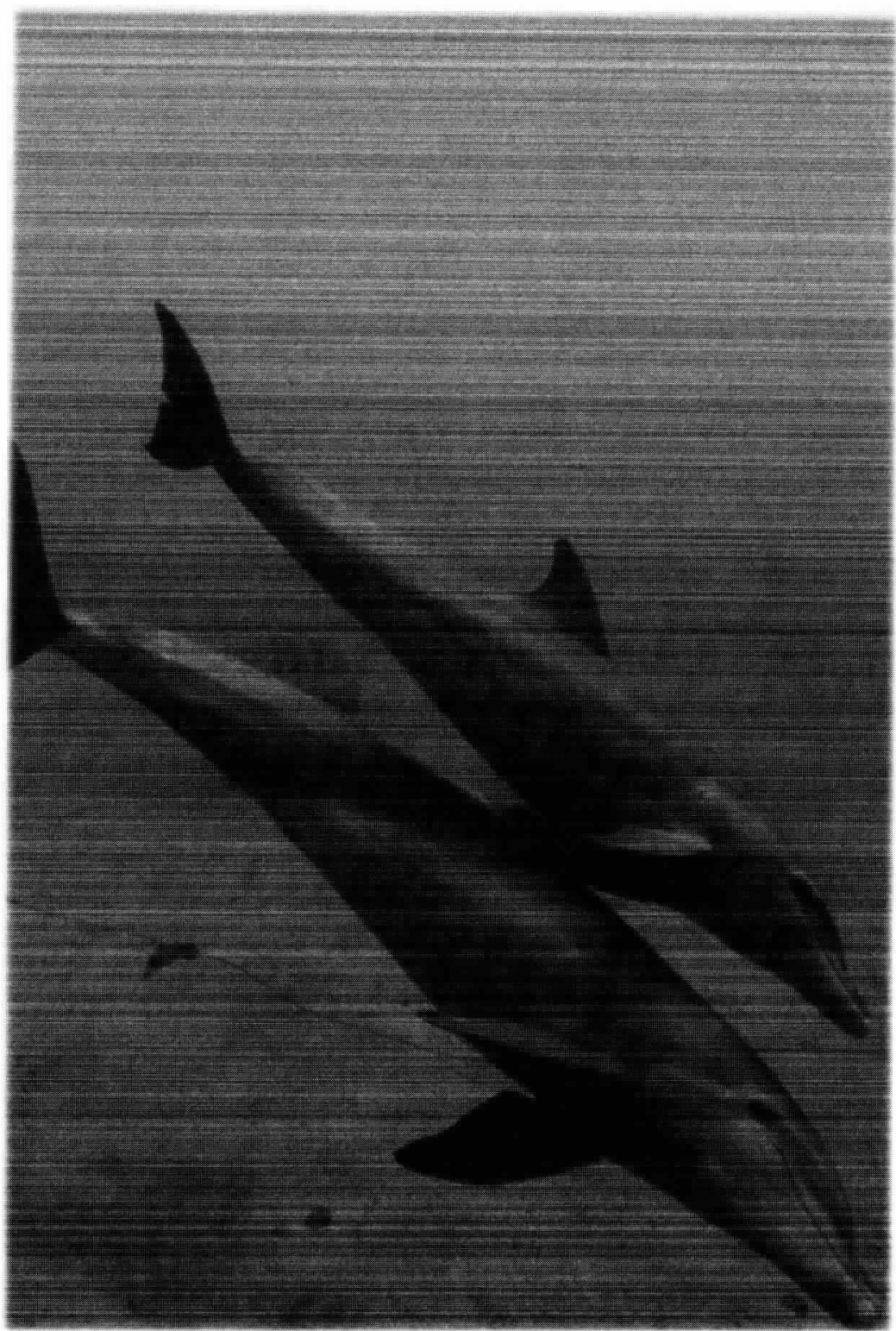

Credit: NOAA Photo Library 